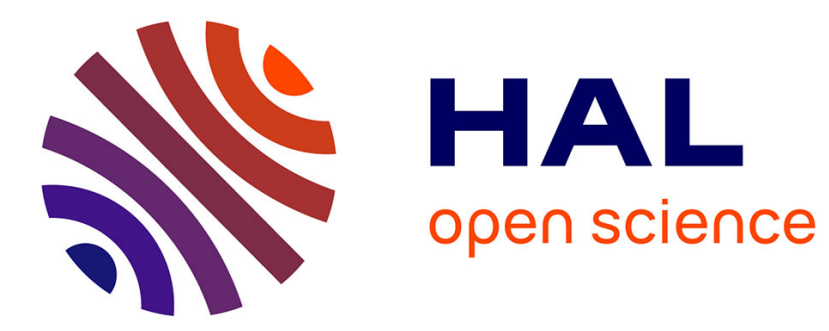

\title{
Causal observability of nonlinear time-delay systems with unknown inputs.
}

Gang Zheng, Jean-Pierre Null Barbot, Driss Boutat, Thierry Floquet, Jean-Pierre Richard

\section{- To cite this version:}

Gang Zheng, Jean-Pierre Null Barbot, Driss Boutat, Thierry Floquet, Jean-Pierre Richard. Causal observability of nonlinear time-delay systems with unknown inputs.. CDC'10, 49th IEEE Conf. on Decision \& Control, Dec 2010, Atlanta, Georgia, United States. hal-00519504

\section{HAL Id: hal-00519504 https://hal.science/hal-00519504}

Submitted on 20 Dec 2010

HAL is a multi-disciplinary open access archive for the deposit and dissemination of scientific research documents, whether they are published or not. The documents may come from teaching and research institutions in France or abroad, or from public or private research centers.
L'archive ouverte pluridisciplinaire HAL, est destinée au dépôt et à la diffusion de documents scientifiques de niveau recherche, publiés ou non, émanant des établissements d'enseignement et de recherche français ou étrangers, des laboratoires publics ou privés. 


\title{
Causal observability of nonlinear time-delay systems with unknown inputs
}

\author{
G. Zheng , J-P. Barbot, D. Boutat, T. Floquet, J.-P. Richard
}

\begin{abstract}
This paper investigates the problem of causal observability of the states and unknown inputs of nonlinear time-delay systems. Using the theory of non-commutative rings, the nonlinear time-delay system is transformed into a suitable canonical form to solve the problem. A necessary and sufficient condition is given to guarantee the existence of a change of coordinate leading to such a form. Then, algorithms are provided to check the possibility of obtaining causal estimations of the states and unknown inputs for the studied systems.
\end{abstract}

\section{INTRODUCTION}

Observation and estimation are important issues in control theory. For linear time-delay systems, various aspects of the observability problem have been studied in the literature, using different methods such as the functional analytic approach ([2]) or the algebraic approach ([3], [18], [6]). The theory of non-commutative rings has been applied to analyze nonlinear time-delay systems firstly in [15] for the disturbance decoupling problem of nonlinear time-delay systems, and for observability of nonlinear time-delay systems with known inputs in [19].

In this algebraic framework, the left Ore ring of noncommutative polynomials defined over the field of meromorphic functions is used for the analysis of nonlinear timedelay systems, since the rank of a module over this ring is well defined and can be used to characterize controllability, observability and identifiability of nonlinear time-delay systems.

Based on the algebraic framework proposed in [19], the problem of causal and non-causal observability of the states and unknown inputs for nonlinear time-delay systems has been studied in [20] assuming that the systems can be transformed into a suitable canonical form. This condition is relaxed in this paper by introducing additional information depending on the available measurements. A necessary and sufficient condition is given to guarantee the existence of those new variables.

G. Zheng is with Project ALIEN, INRIA Lille-Nord Europe, 40, avenue Halley, 59650 Villeneuve d'Ascq, France gang.zheng@inria. fr

J.-P. Barbot is with ECS ENSEA, 6 Avenue du Ponceau, 95014 CergyPontoise, and Project ALIEN, INRIA, France barbot @ensea . fr

D. Boutat is with ENSI de Bourges PRISME, 10 Boulevard de Lahitolle, 18020 Bourges, France driss.boutat@ensi-bourges. fr

T. Floquet and J.-P. Richard are with LAGIS FRE CNRS 3303, Ecole Centrale de Lille, BP 48, 59651 Villeneuve d'Ascq, and Project ALIEN, INRIA, France \{thierry.floquet, jean-pierre.richard\}@ec-lille.fr

\section{AlgEBRAIC FRAMEWORK}

Consider the following nonlinear time-delay system:

$\left\{\begin{array}{l}\dot{x}=f(x(t-i \tau))+\sum_{j=0}^{s} g^{j}(x(t-i \tau)) u(t-j \tau) \\ y=h(x(t-i \tau))=\left[h_{1}(x(t-i \tau)), \ldots, h_{p}(x(t-i \tau))\right]^{T} \\ x(t)=\psi(t), u(t)=\varphi(t), t \in[-s \tau, 0]\end{array}\right.$

where $x \in W \subset R^{n}$ denotes the state variables, $u=$ $\left[u_{1}, \ldots, u_{m}\right]^{T} \in R^{m}$ is the unknown admissible input and $y \in R^{p}$ is the measurable output. Without loss of generality, it can be assumes that $p \geq m$. $\tau$ is the basic time delay, and it is assumed that all times delays are multiple times of $\tau . i \in S_{-}=\{0,1, \ldots, s\}$, a finite set of constant time-delays. $f, g^{j}$ and $h$ are meromorphic functions ${ }^{1}$ where $f(x(t-i \tau))=f(x, x(t-\tau), \ldots, x(t-s \tau)) . \psi:[-s \tau, 0] \rightarrow$ $R^{n}$ and $\varphi:[-s \tau, 0] \rightarrow R^{m}$ denote unknown continuous functions of initial conditions. In this work, it is assumed that the system (1) with $u=0$ is locally observable, and admits a unique solution for initial conditions $\psi$ and $\varphi$.

Based on the algebraic framework introduced in [19], let $\mathcal{K}$ be the field of meromorphic functions of a finite number of the variables from $\left\{x_{j}(t-i \tau), j \in[1, n], i \in S_{-}\right\}$. With the standard differential operator $d$, define the vector space $\mathcal{E}$ over $\mathcal{K}$ :

$$
\mathcal{E}=\operatorname{span}_{\mathcal{K}}\{d \xi: \xi \in \mathcal{K}\}
$$

which is the set of linear combinations of a finite number of one-forms from $d x_{j}(t-i \tau)$ with row vector coefficients in $\mathcal{K}$. Introduce the backward time-shift operator $\delta$ defined by

$$
\delta^{i} \xi(t)=\xi(t-i \tau), \xi(t) \in \mathcal{K}, \text { for } i \in Z^{+}
$$

and

$$
\begin{aligned}
\delta^{i}(a(t) d \xi(t)) & =\delta^{i} a(t) \delta^{i} d \xi(t) \\
& =a(t-i \tau) d \xi(t-i \tau)
\end{aligned}
$$

for $a(t) d \xi(t) \in \mathcal{E}$, and $i \in Z^{+}$.

Let $\mathcal{K}(\delta]$ denote the set of polynomials of the form

$$
a(\delta]=a_{0}(t)+a_{1}(t) \delta+\cdots+a_{r_{a}}(t) \delta^{r_{a}}
$$

where $a_{i}(t) \in \mathcal{K}$. The addition in $\mathcal{K}(\delta]$ is defined as usual, but the multiplication is given by

$$
a(\delta] b(\delta]=\sum_{k=0}^{r_{a}+r_{b}} \sum_{i+j=k}^{i \leq r_{a}, j \leq r_{b}} a_{i}(t) b_{j}(t-i \tau) \delta^{k}
$$

Note that $\mathcal{K}(\delta]$ satisfies the associative law and is a noncommutative ring (see [19]). However, it is proved that the ring $\mathcal{K}(\delta]$ is a left Ore ring [12], [19], which enables to define

\footnotetext{
${ }^{1}$ i.e. quotients of convergent power series with real coefficients [4], [19]
} 
the rank of a module over this ring. Let $\mathcal{M}$ denote the left module over $\mathcal{K}(\delta]: \mathcal{M}=\operatorname{span}_{\mathcal{K}(\delta)}\{d \xi, \xi \in \mathcal{K}\}$, where $\mathcal{K}(\delta]$ acts on $d \xi$ according to (2) and (3).

With the definition of $\mathcal{K}(\delta]$, (1) can be rewritten in a more compact form as follows:

$$
\left\{\begin{array}{l}
\dot{x}=f(x, \delta)+\sum_{i=1}^{m} G_{i} u_{i}(t) \\
y=h(x, \delta) \\
x(t)=\psi(t), u(t)=\varphi(t), t \in[-s \tau, 0]
\end{array}\right.
$$

where $f(x, \delta)=f(x(t-i \tau))$ and $h(x, \delta)=h(x(t-i \tau))$ with entries belonging to $\mathcal{K}, G_{i}=\sum_{j=0}^{s} g_{i}^{j} \delta^{j}$ with entries belonging to $\mathcal{K}(\delta]$. It is assumed that $\operatorname{rank}_{\mathcal{K}(\delta]} \frac{\partial h}{\partial x}=p$, which implies that $\left[h_{1}, \ldots, h_{p}\right]^{T}$ are independent functions of $x$ and its backward time-shifts.

\section{NOTATIONS AND DEFINITIONS}

Observability definitions for nonlinear systems without delays have been given given in [10] and in [5]. A definition of observability for time-delay systems is given in [14]. A more generic definition is stated here as follows:

Definition 1: System (1) is locally observable if the state $x(t) \in W \subset R^{n}$ can be expressed as a function of the output and its derivatives with their backward and forward shifts as follows:

$$
x(t)=\alpha\left(y(t-j \tau), \ldots, y^{(k)}(t-j \tau)\right)
$$

for $j \in Z$ and $k \in Z^{+}$. It is locally causally observable if (7) is satisfied for $j \in Z^{+}$and $k \in Z^{+}$, and locally non-causally observer if (7) is satisfied for $j \in Z$ and $k \in Z^{+}$.

Following the same principle of Definition 1, the following definition for the reconstruction of the unknown inputs is given.

Definition 2: The unknown input $u(t)$ can be estimated if it can be written as a function of the derivatives of the output and its derivatives with backward and forward shifts, i.e.

$$
u(t)=\beta\left(y(t-j \tau), \ldots, y^{(k)}(t-j \tau)\right)
$$

for $j \in Z$ and $k \in Z^{+}$. It can be causally estimated if (8) is satisfied for $j \in Z^{+}$and $k \in Z^{+}$, and non-causally estimated if (8) is satisfied for $j \in Z$ and $k \in Z^{+}$.

Definition 3: (Unimodular matrix) [14] A matrix $A \in$ $\mathcal{K}^{n \times n}(\delta]$ is said to be unimodular over $\mathcal{K}(\delta]$ if it has a left inverse $A^{-1} \in \mathcal{K}^{n \times n}(\delta]$, such that $A^{-1} A=I_{n \times n}$.

Definition 4: (Change of coordinate) [14] $z=\phi(\delta, x) \in$ $\mathcal{K}^{n \times 1}$ is a causal change of coordinate over $\mathcal{K}$ for system (1) if there exist locally a function $\phi^{-1} \in \mathcal{K}^{n \times 1}$ and some constants $c_{1}, \cdots, c_{n} \in N$ such that

$$
\operatorname{diag}\left\{\delta^{c_{i}}\right\} x=\phi^{-1}(\delta, z) .
$$

The change of coordinate is bicausal over $\mathcal{K}$ if $\max \left\{c_{i}\right\}=0$, i.e. $x=\phi^{-1}(\delta, z)$.

Note that the relative degree for nonlinear systems without delays is well defined via the Lie derivative (see [11]). Then many efforts have been done to extend the classical Lie derivative for nonlinear time-delay systems. Several researchers tried to extend the Lie derivative to nonlinear time-delay systems (see [8], [9], [16], [17]) in the framework of commutative rings. In what follows the relative degree and the observability indices for nonlinear time-delay systems are defined by extending the Lie derivative in the algebraic framework of [19], from the non-commutative rings point of view.

Let $f(x(t-j \tau))$ and $h(x(t-j \tau))$ for $0 \leq j \leq s$ be an $n$ and $p$ dimensional vectors, respectively, with entries $f_{r} \in \mathcal{K}$ for $1 \leq r \leq n$ and $h_{i} \in \mathcal{K}$ for $1 \leq i \leq p$. Let

$$
\frac{\partial h_{i}}{\partial x}=\left[\frac{\partial h_{i}}{\partial x_{1}}, \cdots, \frac{\partial h_{i}}{\partial x_{n}}\right] \in \mathcal{K}^{1 \times n}(\delta]
$$

where for $1 \leq r \leq n$ :

$$
\frac{\partial h_{i}}{\partial x_{r}}=\sum_{j=0}^{s} \frac{\partial h_{i}}{\partial x_{r}(t-j \tau)} \delta^{j} \in \mathcal{K}(\delta]
$$

then the Lie derivative for nonlinear systems without delays can be extended to nonlinear time-delay systems in the framework of [19] as follows

$$
L_{f} h_{i}=\frac{\partial h_{i}}{\partial x}(f)=\sum_{r=1}^{n} \sum_{j=0}^{s} \frac{\partial h_{i}}{\partial x_{r}(t-j \tau)} \delta^{j}\left(f_{r}\right)
$$

For $j=0,(10)$ is the classical definition of the Lie derivative of $h$ along $f$. For $h_{i} \in \mathcal{K}$, define

$$
L_{G_{i}} h_{i}=\frac{\partial h_{i}}{\partial x}\left(G_{i}\right)
$$

Using the above definition of Lie derivative, the relative degree can be defined in the following way:

Definition 5: (Relative degree) System (6) has relative degree $\left(\nu_{1}, \cdots, \nu_{p}\right)$ in an open set $W \subseteq R^{n}$ if, for $1 \leq$ $i \leq p$, the following conditions are satisfied :

1) for all $x \in W, L_{G_{j}} L_{f}^{r} h_{i}(x, \delta)=0$, for all $1 \leq j \leq m$ and $0 \leq r<\nu_{i}-1$;

2) there exists $x \in W$ such that $\exists j \in[1, m]$, $L_{G_{j}} L_{f}^{\nu_{i}-1} h_{i}(x, \delta) \neq 0$.

If for $1 \leq i \leq p,(1)$ is satisfied for all $r \geq 0$, then we set $\nu_{i}=\infty$.

Since (6) is assumed to be locally observable when $u=$ 0 , then one can define, for (6), the so-called observability indices introduced in [13]. Let

$$
\mathcal{F}_{k}:=\operatorname{span}_{\mathcal{K}(\delta]}\left\{d h, d L_{f} h, \cdots, d L_{f}^{k-1} h\right\}
$$

for $1 \leq k \leq n$. It can be shown that the filtration of $\mathcal{K}(\delta]$ module satisfies

$$
\mathcal{F}_{1} \subset \mathcal{F}_{2} \subset \cdots \subset \mathcal{F}_{n}
$$

Then define

$$
d_{1}=\operatorname{rank}_{\mathcal{K}(\delta]} \mathcal{F}_{1}
$$

and

$$
d_{k}=\operatorname{rank}_{\mathcal{K}(\delta]} \mathcal{F}_{k}-\operatorname{rank}_{\mathcal{K}(\delta]} \mathcal{F}_{k-1}
$$

for $2 \leq k \leq n$. Let

$$
k_{i}=\operatorname{card}\left\{d_{k} \geq i, 1 \leq k \leq n\right\} .
$$


Then $\left(k_{1}, \cdots, k_{p}\right)$ are the observability indices and

$$
\sum_{i=1}^{p} k_{i}=n
$$

since (6) is locally observable with $u=0$. Reorder, if necessary, the output components of (6), such that

$$
\begin{aligned}
& \operatorname{rank}_{\mathcal{K}(\delta]}\left\{\frac{\partial h_{1}}{\partial x}, \cdots, \frac{\partial L_{f}^{k_{1}-1} h_{1}}{\partial x}, \cdots, \frac{\partial h_{p}}{\partial x}, \cdots, \frac{\partial L_{f}^{k_{p}-1} h_{p}}{\partial x}\right\} \\
& =k_{1}+\cdots+k_{p}=n
\end{aligned}
$$

Since $\operatorname{rank}_{\mathcal{K}(\delta]} \frac{\partial h}{\partial x}=p$, the observability indices $\left(k_{1}, \cdots, k_{p}\right)$ for $\left(h_{1}, \cdots, h_{p}\right)$ are well defined, but the order may be not unique.

\section{CANONICAL FORM AND CAUSAL OBSERVABILITY}

Hereafter are recalled some results given in [20].

Proposition 1: [20] For $1 \leq i \leq p$, denote $k_{i}$ the observability indices and $\nu_{i}$ the relative degree index for $y_{i}$ of (6), and note $\rho_{i}=\min \left\{\nu_{i}, k_{i}\right\}$. Then there exists a change of coordinate $\phi(x, \delta) \in \mathcal{K}^{n \times 1}$, such that (6) can be transformed into the following form:

$$
\begin{aligned}
\dot{z}_{i, j} & =z_{i, j+1} \\
\dot{z}_{i, \rho_{i}} & =V_{i}=L_{f}^{\rho_{i}} h_{i}(x, \delta)+\sum_{j=1}^{m} L_{G_{j}} L_{f}^{\rho_{i}-1} h_{i}(x, \delta) u_{j} \\
y_{i} & =C_{i} z_{i}=z_{i, 1} \\
\dot{\xi} & =\alpha(z, \xi, \delta)+\beta(z, \xi, \delta) u
\end{aligned}
$$

where

$$
\begin{aligned}
z_{i} & =\left(z_{i, 1}, \cdots, z_{i, \rho_{i}}\right)^{T}=\left(h_{i}, \cdots, L_{f}^{\rho_{i}-1} h_{i}\right)^{T} \in \mathcal{K}^{\rho_{i} \times 1} \\
\alpha & \in \mathcal{K}^{\mu \times 1}, \beta \in \mathcal{K}^{\mu \times 1}(\delta] \text { with } \mu=n-\sum_{j=1}^{p} \rho_{j} \\
C_{i} & =(1,0, \cdots, 0) \in R^{1 \times \rho_{i}}
\end{aligned}
$$

Moreover if $k_{i}<\nu_{i}$, one has $V_{i}=L_{f}^{\rho_{i}} h_{i}=L_{f}^{k_{i}} h_{i}$.

For (11), note

$$
H(x, \delta)=\Psi(x, \delta)+\Gamma(x, \delta) u
$$

with

$$
\begin{aligned}
& H(x, \delta)=\left(V_{1}, \cdots, V_{p}\right)^{T} \\
& \Psi(x, \delta)=\left(L_{f}^{\rho_{1}} h_{1}, \cdots, L_{f}^{\rho_{p}} h_{p}\right)^{T}
\end{aligned}
$$

and

$$
\Gamma(x, \delta)=\left(\begin{array}{ccc}
L_{G_{1}} L_{f}^{\rho_{1}-1} h_{1} & \cdots & L_{G_{m}} L_{f}^{\rho_{1}-1} h_{1} \\
\vdots & \ddots & \vdots \\
L_{G_{1}} L_{f}^{\rho_{p}-1} h_{p} & \cdots & L_{G_{m}} L_{f}^{\rho_{p}-1} h_{p}
\end{array}\right)
$$

where $H(x, \delta) \in \mathcal{K}^{p \times 1}, \Psi(x, \delta) \in \mathcal{K}^{p \times 1}$ and $\Gamma(x, \delta) \in$ $\mathcal{K}^{p \times m}(\delta]$. Since $\Gamma \in \mathcal{K}^{p \times m}(\delta]$ with $m \leq p$, if

$$
\operatorname{rank}_{\mathcal{K}(\delta]} \Gamma=m
$$

there exists a matrix $\Xi \in \mathcal{K}^{p \times p}(\delta]$ such that

$$
\Xi \Gamma=\left[\begin{array}{l}
\bar{\Gamma} \\
\mathbf{0}
\end{array}\right]
$$

where $\bar{\Gamma} \in \mathcal{K}^{m \times m}(\delta]$ has full rank $m$. The set $\Phi$ is introduced as follows:

$$
\Phi=\left\{d h_{1}, \cdots, d L_{f}^{\rho_{1}-1} h_{1}, \cdots, d h_{p}, \cdots, d L_{f}^{\rho_{p}-1} h_{p}\right\}
$$

Then the following theorem can be stated.

Theorem 1: [20] Consider the system (6) with outputs $\left(y_{1}, \ldots, y_{p}\right)$ and the corresponding $\left(\rho_{1}, \ldots, \rho_{p}\right)$ with $\rho_{i}=$ $\min \left\{k_{i}, \nu_{i}\right\}$ where $k_{i}$ and $\nu_{i}$ are the observability indices and the relative degree indexes, respectively. If

$$
\operatorname{rank}_{\mathcal{K}(\delta]} \Phi=n
$$

where $\Phi$ is defined in (17), then there exists a change of coordinate $\phi(x, \delta)$ such that (6) can be transformed into (1114) with $\operatorname{dim} \xi=0$.

Moreover, if the change of coordinate is locally bicausal over $\mathcal{K}$, then the state $x(t)$ of (6) is locally causally observable, and if $\bar{\Gamma} \in \mathcal{K}^{m \times m}(\delta]$ is also unimodular over $\mathcal{K}(\delta]$, then the unknown input $u(t)$ of (6) can be causally estimated as well.

Due to the particular triangular structure of the system (11)(12), the whole state of the system, as well as the unknown input, can be estimated in finite time using higher order sliding mode observers [7].

When $\operatorname{rank}_{\mathcal{K}(\delta]} \Phi<n$, the problem is to estimate the state $\xi$. It may be still possible provided that some additional conditions are satisfied. This is detailed in the next Section.

\section{EXTENDED CASE}

In [1], a constructive algorithm to solve the above mentioned problem for nonlinear systems without delays has been studied. The result of this section can be seen as an extension of the work [1] to treat the observation problem for time-delay systems with unknown inputs of the form (6). The objective is to generate additional variables from the available measurement and unaffected by the unknown input such that an extended canonical form similar to (11)-(12) can be obtained for the estimation of the remaining state $\xi$.

For this, consider $\Phi$ defined in (17). If $\operatorname{rank}_{\mathcal{K}(\delta]} \Phi=j$, one can select $j$ linearly independent vectors over $\mathcal{K}(\delta]$ from $\Phi$, denoted as

$$
\Phi=\left\{d z_{1}, \cdots, d z_{j}\right\}
$$

Note

$$
£=\operatorname{span}_{R[\delta]}\left\{z_{1}, \cdots, z_{j}\right\}
$$

where $R[\delta]$ is the commutative ring of polynomials of $\delta$ with coefficients belonging to the field $R$ and let $£(\delta]$ be the set of polynomials of $\delta$ with coefficients over $£$. Define the module spanned by the elements of $\Phi$ over $£(\delta]$ as follows

$$
\Omega=\operatorname{span}_{£(\delta]}\{\xi, \xi \in \Phi\} .
$$

Define also

$$
\mathcal{G}=\operatorname{span}_{R[\delta]}\left\{G_{1}, \ldots, G_{m}\right\}
$$

and its left annihilator

$$
\mathcal{G}^{\perp}=\operatorname{span}_{R[\delta]}\{\omega \in \Omega \mid \omega g=0, \forall g \in \mathcal{G}\} .
$$


Based on the above definitions, let us state the main result of the paper.

Theorem 2: Consider the system (6) with outputs $y=$ $\left(y_{1}, \cdots, y_{p}\right)^{T}$ and the corresponding $\left(\rho_{1}, \ldots, \rho_{p}\right)$ with $\rho_{i}=$ $\min \left\{k_{i}, \nu_{i}\right\}$ where $k_{i}$ and $\nu_{i}$ are the observability indices and the relative degree indexes, respectively. Suppose $\operatorname{rank}_{\mathcal{K}(\delta]} \Phi<n$ where $\Phi$ is defined in (17). There exist $l$ new independent outputs over $\mathcal{K}$ suitable to the causal estimation problem if and only if

$$
\operatorname{rank}_{\mathcal{K}} \mathcal{H}=l
$$

where

$$
\mathcal{H}=\operatorname{span}_{R[\delta]}\left\{\omega \in \mathcal{G}^{\perp} \cap \Omega \mid \omega f \notin £\right\} .
$$

Moreover, the $l$ additional outputs, denoted $\bar{y}_{i}, 1 \leq i \leq l$, are given by:

$$
\bar{y}_{i}=\omega_{i} f \quad \bmod £
$$

where $\omega_{i} \in \mathcal{H}$.

\section{Proof:}

Denote $Q_{i}=\left[q_{1}^{i}, \cdots, q_{p}^{i}\right]$ as a $1 \times p$ vector with $q_{j}^{i} \in \mathcal{K}(\delta]$ for $1 \leq j \leq p$. One has

$$
\begin{aligned}
Q_{i} \Gamma= & Q_{i}\left(\begin{array}{ccc}
L_{G_{1}} L_{f}^{\rho_{1}-1} h_{1} & \cdots & L_{G_{m}} L_{f}^{\rho_{1}-1} h_{1} \\
\vdots & \ddots & \vdots \\
L_{G_{1}} L_{f}^{\rho_{p}-1} h_{p} & \cdots & L_{G_{m}} L_{f}^{\rho_{p}-1} h_{p}
\end{array}\right) \\
= & \left(Q_{i}\left[\begin{array}{c}
\frac{\partial L_{f}^{\rho_{1}-1} h_{1}}{\partial x} \\
\vdots \\
\frac{\partial L_{f}^{\rho_{p}-1} h_{p}}{\partial x}
\end{array}\right]\right)\left[G_{1}, \cdots, G_{m}\right]
\end{aligned}
$$

because of the associativity law over $\mathcal{K}(\delta]$. Then according to the definition (9), one gets

$$
Q_{i} \Gamma=\omega_{i}\left[G_{1}, \cdots, G_{m}\right]=\omega_{i} G
$$

where $\omega_{i}=\sum_{c=1}^{n} \sum_{j=1}^{p} q_{j}^{i} \frac{\partial L_{f}^{\rho_{j}-1} h_{j}}{\partial x_{c}} d x_{c}$.

Moreover, one can check that

$$
\begin{aligned}
& \omega_{i} f=\left(Q_{i}\left[\begin{array}{c}
\frac{\partial L_{f}^{\rho_{1}-1} h_{1}}{\partial x} \\
\vdots \\
\frac{\partial L_{f}^{\rho_{p}-1} h_{p}}{\partial x}
\end{array}\right]\right) f \\
& =Q_{i}\left(\left[\begin{array}{c}
\frac{\partial L_{f}^{\rho_{1}-1} h_{1}}{\partial x} \\
\vdots \\
\frac{\partial L_{f}^{\rho_{p}-1} h_{p}}{\partial x}
\end{array}\right] f\right) \\
& =Q_{i}\left[\begin{array}{c}
L_{f}^{\rho_{1}} h_{1} \\
\vdots \\
L_{f}^{\rho_{p}} h_{p}
\end{array}\right]=Q_{i} \Psi .
\end{aligned}
$$

According to (15), one has

$$
Q_{i} H=Q_{i}(\Psi+\Gamma u)=\omega_{i} f+\omega_{i} G u
$$

where $H=\left(V_{1}, \cdots, V_{p}\right)^{T}$ is a vector which can be estimated in finite time but which is affected by the unknown input. Thus, one can generate $l$ additional information suitable to solve the estimation problem if and only if one can find $l$ independent $\left\{Q_{1}, \cdots, Q_{l}\right\}$ vectors over $\mathcal{K}(\delta]$ such that for each $Q_{i}=\left\{q_{1}^{i}, \cdots, q_{p}^{i}\right\}$ satisfying $q_{j}^{i} \in £(\delta]$, one has $Q_{i} \Gamma=0$ and $Q_{i} H \notin £$,

Thus, one has to prove that the following conditions are equivalent:

1) there exist $l$ row vectors $Q_{i}=\left[q_{1}^{i}, \cdots, q_{p}^{i}\right]$, with $q_{j}^{i} \in$ $£(\delta]$, such that $\operatorname{rank}_{\mathcal{K}(\delta]}\left\{Q_{1}, \cdots, Q_{l}\right\}=l, Q_{i} \Gamma=0$ and $Q_{i} H \notin £$.

2) $\operatorname{rank}_{\mathcal{K}} \mathcal{H}=l$ with $\mathcal{H}$ defined in (19).

Necessity: Suppose item 1) is satisfied, then according to (20), one has

$$
Q_{i} \Gamma=\omega_{i} G=0 \Rightarrow \omega_{i} \in \mathcal{G}^{\perp}
$$

and

$$
Q_{i} H=\omega_{i} f \notin £ .
$$

Thus $\operatorname{rank}_{\mathcal{K}(\delta]}\left\{Q_{1}, \cdots, Q_{l}\right\}=l$ implies $\operatorname{rank}_{\mathcal{K}}\left\{\omega_{1}, \cdots, \omega_{l}\right\}=l$.

Since $L_{f}^{\rho_{i}-1} h_{i} \in £$ and $q_{i} \in £(\delta]$, then $\omega_{i}=$ $\sum_{c=1}^{n} \sum_{j=1}^{p} q_{j}^{i} \frac{\partial L_{f}^{\rho_{j}-1} h_{j}}{\partial x_{c}} d x_{c} \in \Omega$. Thus, $\omega_{i} \in \mathcal{H}$ defined in (19).

Sufficiency: Suppose $\operatorname{rank}_{\mathcal{K}} \mathcal{H}=l$. Then one can find $l$ independent 1 -forms over $\mathcal{K}:\left\{\omega_{1}, \cdots, \omega_{l}\right\}$ with $\omega_{i} \in$ $\mathcal{G}^{\perp} \cap \Omega$ which implies there exist $l$ independent vectors over $\mathcal{K}(\delta]:\left\{Q_{1}, \cdots, Q_{l}\right\}$ with entries belonging to $£(\delta]$ such that $\operatorname{rank}_{\mathcal{K}(\delta]}\left\{Q_{1}, \cdots, Q_{l}\right\}=l$, since for each $Q_{i}$ one has

$$
Q_{i} \Gamma=\omega_{i} G=0
$$

and

$$
Q_{i} H=\omega_{i} f \notin £
$$

The variable

$$
\bar{y}_{i}=Q_{i} H=\omega_{i} f \quad \bmod £
$$

can be used as an additional output since

1) $H$ can be estimated in finite time;

2) $Q_{i}$ has entries in $£(\delta]$;

3) $\bar{y}_{i}$ do not belong to the current set $£$ of measured variables.

Theorem 2 means that if $\operatorname{rank}_{\mathcal{K}} \mathcal{H}=l$, one can define an extended output $\left[y^{T}, \bar{y}\right]^{T} \in \mathbb{R}^{p+l}$ with observability indices $\bar{k}_{i}$ and relative degree indexes $\bar{\nu}_{i}$. Then, similarly to (17), a new $\bar{\Phi}$ can be defined. If $\operatorname{rank}_{\mathcal{K}(\delta]} \bar{\Phi}=n$, the state and the unknown inputs can be estimated in finite time. Otherwise, if $\operatorname{rank}_{\mathcal{K}(\delta]} \bar{\Phi}<n$ and if Theorem 2 is still valid, the procedure is iterated until Theorem 1 is true, resulting in the causal observation of the states and unknown inputs for nonlinear time-delay systems of the form (6).

In the tables Algorithm 1 and 2, two algorithms are proposed in order to check the possible causal estimation of the states and unknown inputs. Algorithm 2 is used to determine whether the studied system can be transformed into the proposed canonical form, by using Theorem 2 . If it 
is, Algorithm 1 then checks whether the states and unknown inputs of the system are causally observable.

Let us note that it is the locally bicausal change of coordinate which makes the state of system locally causally observable. And it is the unimodular characteristic of $\Gamma$ over $\mathcal{K}(\delta]$ which guarantees the causal reconstruction of unknown inputs. The non-causal case was studied in [20].

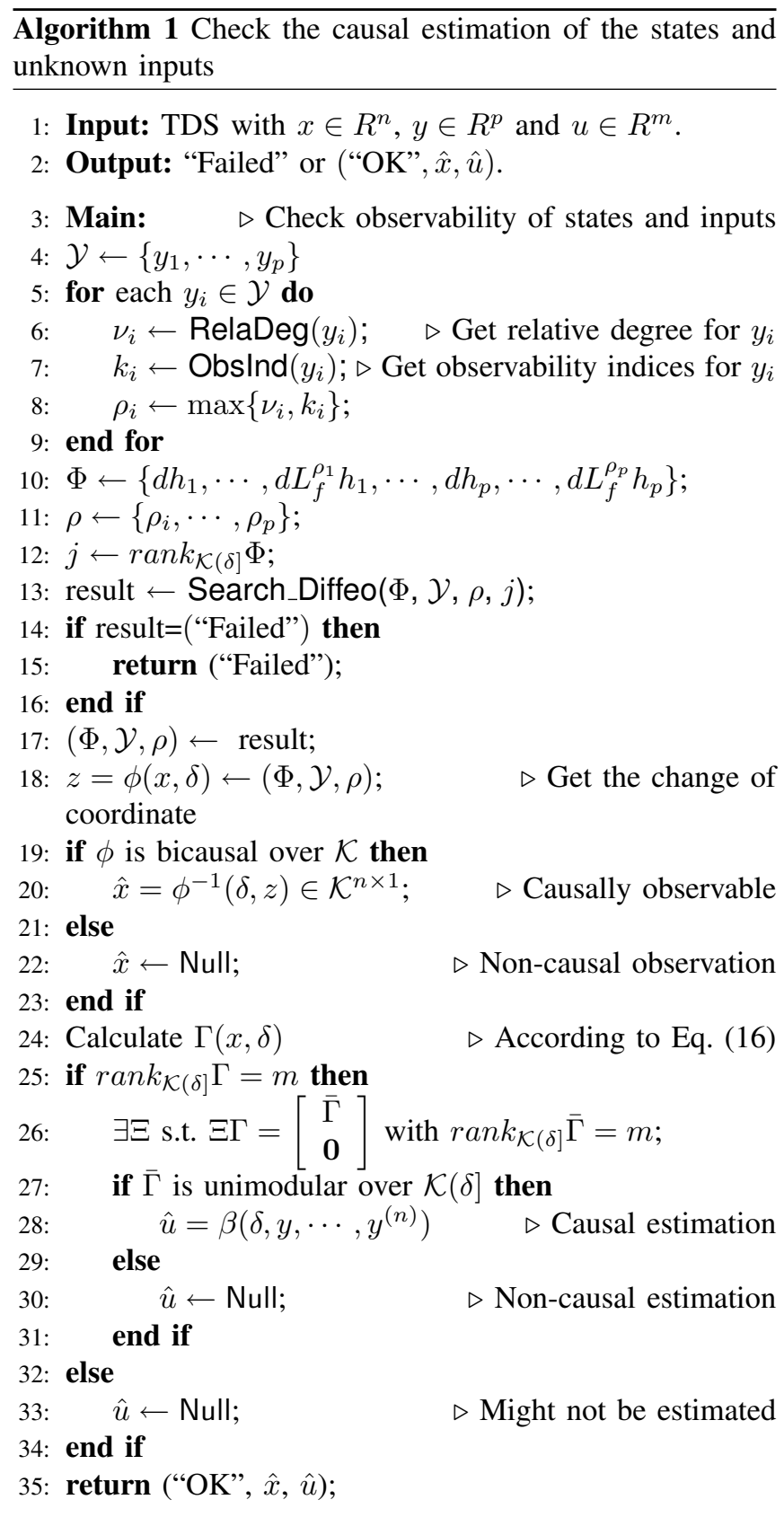

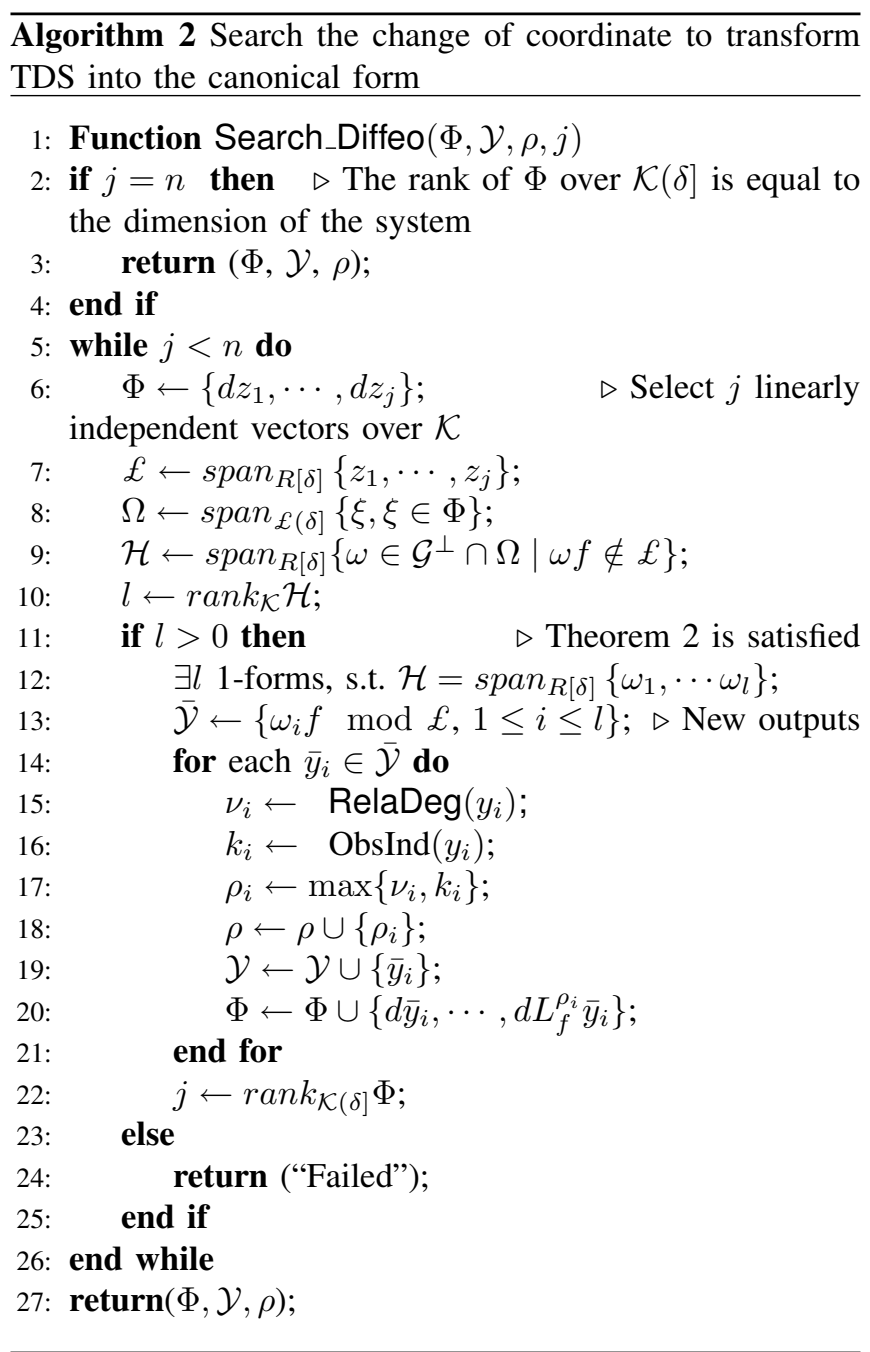

\section{ILLUSTRATIVE EXAMPLE}

Consider the following dynamical system:

$$
\left\{\begin{array}{l}
\dot{x}_{1}=-\delta x_{1}+\delta x_{4} u_{1} \\
\dot{x}_{2}=-\delta x_{3}+x_{4} \\
\dot{x}_{3}=x_{2}-\delta x_{4} u_{1} \\
\dot{x}_{4}=u_{2} \\
y_{1}=x_{1} \\
y_{2}=\delta x_{1}+x_{3}
\end{array}\right.
$$

One can check that

$$
\rho_{1}=\rho_{2}=1
$$

and $\Phi=\left\{d x_{1}, \delta d x_{1}+d x_{3}\right\}$ whichgivesrank $k_{\mathcal{K}(\delta]} \Phi=2<$ $n$.

$$
\begin{aligned}
& \text { Set } \mathcal{G}=\operatorname{span}_{R[\delta]}\left\{G_{1}, \ldots, G_{m}\right\} \text {, one has } \\
& \qquad \mathcal{G}^{\perp}=\operatorname{span}_{R[\delta]}\left\{d x_{1}+d x_{3}, d x_{2}\right\}
\end{aligned}
$$

Since $\operatorname{rank}_{\mathcal{K}(\delta]} \Phi=2, £=\operatorname{span}_{R[\delta]}\left\{x_{1}, \delta x_{1}+x_{3}\right\}$ and

$$
\Omega=\operatorname{span}_{£(\delta]}\{\xi, \xi \in \Phi\}=\operatorname{span}_{£(\delta]}\left\{d x_{1}, d x_{3}\right\} .
$$


One obtains

$$
\begin{aligned}
\Omega \cap \mathcal{G}^{\perp}= & \operatorname{span}_{£(\delta]}\left\{d x_{1}, d x_{3}\right\} \\
& \cap \operatorname{span}_{R[\delta]}\left\{d x_{1}+d x_{3}, d x_{2}\right\} \\
= & \operatorname{span}_{£(\delta]}\left\{d x_{1}+d x_{3}\right\}
\end{aligned}
$$

From the definition of $\mathcal{H}$ in (19), one can check that $\operatorname{rank}_{\mathcal{K}} \mathcal{H}=1$, which gives the one-form $\omega=d x_{1}+d x_{3}$, satisfying $\omega \in \Omega \cap \mathcal{G}^{\perp}$ and $\omega f=-\delta x_{1}+x_{2} \notin £$. Thus, a new output $\bar{y}_{1}=h_{3}$ is given by

$$
\begin{aligned}
\bar{y}_{1} & =h_{3}=\omega f \quad \bmod £ \\
& =x_{2}=\delta y_{1}+(1-\delta) \dot{y}_{1}+\dot{y}_{2}
\end{aligned}
$$

which yields $\nu_{3}=2$ and $k_{3}=2$, thus $\rho_{3}=2$. Finally one has the new $\Phi$ as follows:

$$
\Phi=\left\{d x_{1}, \delta d x_{1}+d x_{3}, d x_{2},-\delta d x_{3}+d x_{4}\right\}
$$

It can be checked that $\operatorname{rank}_{\mathcal{K}(\delta]} \Phi=4$, and the new $£$ is

$$
£=\operatorname{span}_{R[\delta]}\left\{x_{1}, \delta x_{1}+x_{3}, x_{2},-\delta x_{3}+x_{4}\right\}
$$

This gives the following change of coordinate

$$
z=\phi(x, \delta)=\left(x_{1}, \delta x_{1}+x_{3}, x_{2},-\delta x_{3}+x_{4}\right)^{T}
$$

it is easy to check that it is bicausal over $\mathcal{K}(\delta]$, since

$$
x=\phi^{-1}=\left(z_{1}, z_{3}, z_{2}-\delta z_{1}, z_{4}+\delta z_{2}-\delta^{2} z_{1}\right)^{T}
$$

and one gets

$$
\left\{\begin{array}{l}
x_{1}=y_{1} \\
x_{2}=y_{3} \\
x_{3}=y_{2}-\delta y_{1} \\
x_{4}=-\delta^{2} y_{1}+\delta y_{2}+\dot{y}_{3}
\end{array}\right.
$$

where the new output $y_{3}$ is defined in (22).

Moreover, the matrix $\Gamma$ with the new output $y_{3}$ is

$$
\Gamma=\left(\begin{array}{cc}
\delta x_{4} & 0 \\
\delta^{2} x_{4} \delta-\delta x_{4} & 0 \\
\delta^{2} x_{4} \delta & 1
\end{array}\right)
$$

and

$$
\operatorname{rank}_{\mathcal{K}(\delta]} \Gamma=2 .
$$

One can find matrices

$$
\Xi=\left(\begin{array}{lll}
1 & 0 & 0 \\
0 & 0 & 1 \\
0 & 0 & 0
\end{array}\right)
$$

and

$$
\bar{\Gamma}^{-1}=\left(\begin{array}{cc}
\frac{1}{\delta x_{4}} & 0 \\
-\delta & 1
\end{array}\right)
$$

such that

$$
\left[\begin{array}{ll}
\bar{\Gamma}^{-1} & \mathbf{0}
\end{array}\right] \Xi \Gamma=I_{2 \times 2}
$$

Consequently, according to Theorem 1, $u_{1}$ and $u_{2}$ are causally observable. A straightforward computation yields

$$
\left\{\begin{array}{l}
u_{1}=\frac{\dot{y}_{1}}{-\delta^{3} y_{1}+\delta^{2} y_{2}+\delta \dot{y}_{3}} \\
u_{2}=-\delta \dot{y}_{1}+\ddot{y}_{3}
\end{array}\right.
$$

\section{CONCLUSION}

In this paper, causal observation of nonlinear time-delay systems with unknown inputs has been studied. The system has to be transformed in a canonical form suitable for the design of a finite time observer. A necessary and sufficient condition for the existence of a change of coordinate has been given. A constructive algorithm has been proposed to check the possibility to obtain causal estimations of the states and unknown inputs for the studied systems.

\section{REFERENCES}

[1] J.-P. Barbot, D. Boutat, and T. Floquet, "An observation algorithm for nonlinear systems with unknown inputs," Automatica, vol. 45, pp. 1970-1974, 2009.

[2] K. Bhat and H. Koivo, "Modal characterizations of controllability and observability in time delay systems," IEEE Transactions on Automatic Control, vol. 21, no. 2, pp. 292- 293, 1976.

[3] J. Brewer, J. Bunce, and F. V. Vleck, "Linear systems over commutative rings," Marcel Dekker, New York, 1986.

[4] G. Conte, C. Moog, and A. Perdon, "Nonlinear control systems: An algebraic setting," Lecture Notes in Control and Information Sciences, vol. 242, pp. Springer-Verlag,London, 1999.

[5] S. Diop and M. Fliess, "Nonlinear observability, identifiability and persistent trajectories," in Proc. of 36th IEEE Conf. on Decision and Control, 1991.

[6] M. Fliess and H. Mounier, "Controllability and observability of linear delay systems: an algebraic approach," ESAIM: Control, Optimisation and Calculus of Variations, vol. 3, pp. 301-314, 1998.

[7] T. Floquet and J.-P. Barbot, "Super twisting algorithm based stepby-step sliding mode observers for nonlinear systems with unknown inputs," International Journal of Systems Science, vol. 38, pp. 803$815,2007$.

[8] A. Germani, C. Manes, and P. Pepe, "An asymptotic state observer for a class of nonlinear delay systems," Kybernetika, vol. 37, no. 4, pp. 459-478, 2001.

[9] _ "A new approach to state observation of nonlinear systems with delayed output," IEEE Transactions on Automatic Control, vol. 47, no. 1, pp. 96-101, 2002.

[10] R. Hermann and A. Krener, "Nonlinear controllability and observability," IEEE Transactions on Automatic Control, vol. 22, no. 5, pp. 728-740, 1977.

[11] A. Isidori, "Nonlinear control systems (3rd edition)," London: Springer-Verlag, 1995.

[12] J. Ježek, "Rings of skew polynomials in algebraical approach to control theory," Kybernetika, vol. 32, no. 1, pp. 63-80, 1996.

[13] A. Krener, "( $\left.\mathrm{A} d_{f, g}\right),\left(a d_{f, g}\right)$ and locally $\left(a d_{f, g}\right)$ invariant and controllability distributions," SIAM Journal on Control and Optimization, vol. 23, no. 4, pp. 523-549, 1985.

[14] L. Marquez-Martinez, C. Moog, and V. Martin, "Observability and observers for nonlinear systems with time delays," Kybernetika, vol. 38 , no. 4, pp. 445-456, 2002.

[15] C. Moog, R. Castro-Linares, M. Velasco-Villa, and L. A. MarqueMartinez, "The disturbance decoupling problem for time-delay nonlinear systems," IEEE Transactions on Automatic Control, vol. 45, no. 2,2000

[16] T. Oguchi and J.-P. Richard, "Sliding-mode control of retarded nonlinear systems via finite spectrum assignment approach," IEEE Transactions on Automatic Control, vol. 51, no. 9, pp. 1527-1531, 2006.

[17] T. Oguchi, A. Watanabe, and T. Nakamizo, "Input-output linearization of retarded non-linear systems by using an extension of lie derivative," International Journal of Control, vol. 75, no. 8, pp. 582-590, 2002.

[18] E. Sontag, "Linear systems over commutative rings: A survey," Ricerche di Automatica, vol. 7, pp. 1-34, 1976.

[19] X. Xia, L. Marquez, P. Zagalak, and C. Moog, "Analysis of nonlinear time-delay systems using modules over non-commutative rings," Automatica, vol. 38, pp. 1549-1555, 2002.

[20] G. Zheng, J.-P. Barbot, D. Boutat, T. Floquet, and J.-P. Richard, "On finite time observation of nonlinear time-delays systems," in Proc. of the 8th IFAC Symposium on Nonlinear Control Systems, 2010. 